\title{
Plane Symmetric Solutions to the Nonlinear Spinor Field Equations in General Relativity Theory
}

\author{
A. Adomou ${ }^{1,2 *}$, Jonas Edou ${ }^{1}$, Siaka Massou ${ }^{1}$ \\ ${ }^{1}$ Department of Theoretical Physics and Mathematics, University of Abomey-Calavi, Abomey-Calavi, Benin \\ ${ }^{2}$ National Superior Institute of Industrial Technology, INSTI-Lokossa, National University of Sciences, Technology, Engineering \\ and Mathematics of Abomey, Abomey, Benin \\ Email: *elietteadomou@gmail.com
}

How to cite this paper: Adomou, A. Edou, J. and Massou, S. (2019) Plane Symmetric Solutions to the Nonlinear Spinor Field Equations in General Relativity Theory. Journal of Modern Physics, 10, 1222-1234. https://doi.org/10.4236/jmp.2019.1010081

Received: June 7, 2019

Accepted: September 15, 2019

Published: September 18, 2019

Copyright ( 2019 by author(s) and Scientific Research Publishing Inc. This work is licensed under the Creative Commons Attribution International License (CC BY 4.0).

http://creativecommons.org/licenses/by/4.0/

\begin{abstract}
We have obtained exact static plane-symmetric solutions to the spinor field equations with nonlinear terms which are arbitrary functions of invariant $I_{p}=P^{2}=\left(i \bar{\psi} \gamma^{5} \psi\right)^{2}$, taking into account their own gravitational field. It is shown that the initial set of the Einstein and spinor field equations with a power-law nonlinearity have regular solutions with a localized energy density of the spinor field only if $m=0$ ( $m$ is the mass parameter in the spinor field equations). Equations with power and polynomial nonlinearities are studied in detail. In this case, a soliton-like configuration has negative energy. We have also obtained exact static plane-symmetric solutions to the above spinor field equations in flat space-time. It is proved that in this case soliton-like solutions are absent.
\end{abstract}

\section{Keywords}

Lagrangian, Static Plane-Symmetric Metric, Field Equations, Energy-Momentum Tensor, Charge Density, Current Vector, Soliton-Like Solution

\section{Introduction}

The consideration of the nonlinear generalization of classical field theory is the only possibility to overcome the shortcomings to the theory which considers elementary particles as materials points. Indeed, with this theory, it is impossible to get a finite quantity of mass, charge and spin of elementary particles as prouved experimentaly. In order to describe the elemetary particles, it is necessary 
to take account the nonlinear terms in the field equations and their own gravitational field. In these conditions, we obtain the soliton-like solutions which are used in the formation of the fields configurations of elementary particles with limited total energy and localized energy density. The concept of soliton is more used in pure science for different purpose. It's studied by many authors in the gravitational theory. G.N. Shikin has investigated the basics of soliton theory in general relativity. In his work, he has formulated clearly the requirements to be fulfilled by soliton-like solutions [1]. In a remarkable paper on the soliton, A. Adomou and G. N. Shikin have obtained exact plane-symmetric solutions to the spinor field equations with nonlinear terms which are arbitrary functions of the invariant $S=\bar{\psi} \psi$, taking into account their own gravitational field. They have studied in detail equations with power and polynomial nonlinearities. They have shown that the initial set of the Einstein and spinor field equations with a powerlaw nonlinearity has regular solutions with a localized energy density of the spinor field only in the case of zero mass parameter in the spinor field, with a negative energy for the soliton-like configuration. They have also proved that the spinor field equation with a polynomial nonlinearity has a regular solution with positive energy. Their study has come out onto the non existence of soliton-like solutions in the flat space-time [2].

The plane-symmetric solitons of spinor and scalar fiels are studied by B. Saha and G. N. Shikin. They have considered a system of nonlinear spinor and scalar fields with minimal coupling in general relativity. The nonlinear term in the spinor field is given by an arbitrary function depending on the bilinear spinor forms $S=\bar{\psi} \psi$ and $P=\left(i \bar{\psi} \gamma^{5} \psi\right)$. As for the scalar lagrangian, it is chosen as an arbitrary function of the scalar invariant $\Omega=\phi_{, \alpha} \phi^{\alpha}$ that becomes linear when $\Omega \rightarrow 0$. The spinor and scalar fields in question interact with each other by means of a gravitational field. The gravitational field is given by a planesymmetric metric. They have obtained exact plane-symmetric solutions to the gravitational, spinor and scalar field equations. They have also investigated the role of gravitational field in the formation of the configurations with limited total energy,spin and charge. In general, they have proved that the choice of spinor field nonlinearity can lead to the elimination of scalar field contribution to the metric functions, but having it contribution to the total energy unaltered [3]. V. Adanhoum, A. Adomou, F. P. Codo and M. N. Hounkonnou have obtained spherical-symmetric soliton-like solutions of nonlinear spinor field equations in gravitational theory. The regularity properties of the obtained solutions as well as the asymptotic behavior of the energy and charge densities are studied [4].

The aim of the paper is to study the role of nonlinear spinor as well as the own gravitational field in the formation of configurations of elementary particles with localized energy density and limited total energy. In this optic, we choose the nonlinear terms in the spinor field equations which are arbitrary functions of $I_{P}=P^{2}=\left(i \bar{\psi} \gamma^{2} \psi\right)^{2}$.

The rest of the present research work is organized as follows. Section 2 deals 
with fundamental equations and general solutions. In the first time, we have established gravitational and spinor fields equations, using the variational principle and usual algebraic manipulations. in the second time, we have obtained and analized the general solutions of the basic equations established. In Section 3, we have investgated in detail the polynomial nonlinearities equations. Section 4 addresses to the concluding remarks.

\section{Fundamental Equations and Their General Solutions}

The lagrangian of the nonlinear spinor and gravitational fields can be written in the form [3]:

$$
L=\frac{R}{2 \chi}+L_{S p}=\frac{R}{2 \chi}+\frac{i}{2}\left(\bar{\psi}^{\mu} \nabla_{\mu} \psi-\nabla_{\mu} \bar{\psi} \gamma^{\mu} \psi\right)-m \bar{\psi} \psi+L_{N},
$$

with $R$ the scalar curvature and $\chi$ the Einstein's gravitational constant. The nonlinear term $L_{N}$ in spinor lagrangian characterizes the self-interaction of a spinor field. $L_{N}=F\left(I_{p}\right)$ represents an arbitrary function depending on the invariant $I_{p}=\left(i \bar{\psi} \gamma^{5} \psi\right)^{2}$.

The metric of space-time admitting static plane-symmetric may be written as [1]:

$$
\mathrm{d} s^{2}=\mathrm{e}^{2 \gamma} \mathrm{d} t^{2}-\mathrm{e}^{2 \alpha} \mathrm{d} x^{2}-\mathrm{e}^{2 \beta}\left(\mathrm{d} y^{2}+\mathrm{d} z^{2}\right)
$$

Here the speed of light $C$ is taken to be unity and the metric functions $\alpha, \beta, \gamma$ depend exclusively on the spatial variable $x$. They satisfy the coordinate condition having the form:

$$
\alpha=2 \beta+\gamma .
$$

The general form of Einstein equation is:

$$
G_{\mu}^{v}=R_{\mu}^{v}-\frac{1}{2} \delta_{\mu}^{v} R=-\chi T_{\mu}^{v},
$$

where $G_{\mu}^{v}$ is the Einstein's tensor; $R_{\mu}^{v}$ is the Ricci's tensor; $\delta_{\mu}^{v}$ is the Kronecker's symbol and $T_{\mu}^{v}$ is the energy-momentum tensor.

Taking into account the metric tensor $g_{\mu \nu}$, the larangian (1) and the variational principle, we obtain Einstein's field equations for the metric (2) under the coordinate condition (3) [5] [6]:

$$
\begin{gathered}
G_{0}^{0}=\mathrm{e}^{-2 \alpha}\left(2 \beta^{\prime \prime}-2 \gamma^{\prime} \beta^{\prime}-\beta^{\prime 2}\right)=-\chi T_{0}^{0}, \\
G_{1}^{1}=\mathrm{e}^{-2 \alpha}\left(2 \beta^{\prime} \gamma^{\prime}+\beta^{\prime 2}\right)=-\chi T_{1}^{1}, \\
G_{2}^{2}=\mathrm{e}^{-2 \alpha}\left(\beta^{\prime \prime}+\gamma^{\prime \prime}-2 \beta^{\prime} \gamma^{\prime}-\beta^{\prime 2}\right)=-\chi T_{2}^{2}, \\
G_{2}^{2}=G_{3}^{3}, \quad T_{2}^{2}=T_{3}^{3} .
\end{gathered}
$$

Let us write down the spinor field equations for the functions $\psi$ and $\bar{\psi}$ [2] [6]:

$$
\begin{gathered}
i \gamma^{\mu} \nabla_{\mu} \psi-m \psi+F^{\prime}\left(I_{p}\right) \psi=0, \\
i \nabla_{\mu} \bar{\psi} \gamma^{\mu}+m \bar{\psi}-F^{\prime}\left(I_{p}\right) \bar{\psi}=0,
\end{gathered}
$$


with

$$
F^{\prime}\left(I_{p}\right)=2 i P \frac{\partial F}{\partial I_{p}} \gamma^{5}
$$

The metric energy-momentum tensor of the spinor field is [5] [6]

$$
T_{\mu}^{v}=\frac{i}{4} g^{v \rho}\left(\bar{\psi} \gamma_{\mu} \nabla_{\nu} \psi+\bar{\psi} \gamma_{\nu} \nabla_{\mu} \psi-\nabla_{\mu} \bar{\psi} \gamma_{\nu} \psi-\nabla_{\nu} \bar{\psi} \gamma_{\mu} \psi\right)-\delta_{\mu}^{v} L_{S p}
$$

where $L_{s p}$ with respect to (9) and (10) takes the form

$$
L_{S p}=-\frac{1}{2}\left(\bar{\psi} \frac{\partial L_{N}}{\partial \bar{\psi}}+\frac{\partial L_{N}}{\partial \psi} \psi\right)+L_{N}=-2 I_{P} \frac{\partial F\left(I_{p}\right)}{\partial I_{p}}+F\left(I_{p}\right) .
$$

From (13), let us write explicitly the nonzero components of the tensor $T_{\mu}^{v}$ :

$$
\begin{gathered}
T_{0}^{0}=T_{2}^{2}=T_{3}^{3}=-L_{S p}=2 I_{p} \frac{\partial F\left(I_{p}\right)}{\partial I_{p}}-F\left(I_{p}\right) \\
T_{1}^{1}=\frac{i}{2}\left(\bar{\psi} \gamma^{1} \nabla_{1} \psi-\nabla_{1} \bar{\psi} \gamma^{1} \psi\right)+2 I_{p} \frac{\partial F\left(I_{p}\right)}{\partial I_{p}}-F\left(I_{p}\right) .
\end{gathered}
$$

In (9), (10) and (12), $\nabla_{\mu}$ denotes the covariant derivative of spinor, having the form [3] [4] [7] [8]

$$
\nabla_{\mu} \psi=\frac{\partial \psi}{\partial x^{\mu}}-\Gamma_{\mu} \psi \text { or } \nabla_{\mu} \bar{\psi}=\frac{\partial \bar{\psi}}{\partial x^{\mu}}+\Gamma_{\mu} \bar{\psi}
$$

where $\Gamma_{\mu}$ are the spinor affine connection matrices.

In curved space-time, the Dirac's matrices $\gamma^{\mu}$ are defined in the following way.

Using the egalities

$$
\begin{gathered}
g_{\mu v}(x)=e_{\mu}^{a}(x) e_{\nu}^{b}(x) \eta_{a b}, \\
\gamma_{\mu}(x)=e_{\mu}^{a}(x) \bar{\gamma}_{a},
\end{gathered}
$$

where $\eta_{a b}=\operatorname{diag}(1,-1,-1,-1), \bar{\gamma}_{a}$ are Dirac's matrices in flat space-time, $e_{\mu}^{a}(x)$ are tetradic 4-vectors, we obtain:

$$
\gamma^{0}=\mathrm{e}^{-\gamma} \bar{\gamma}^{0}, \quad \gamma^{1}(x)=\mathrm{e}^{-\alpha} \bar{\gamma}^{1}, \quad \gamma^{2}=\mathrm{e}^{-\beta} \bar{\gamma}^{2}, \quad \gamma^{3}=\mathrm{e}^{-\beta} \bar{\gamma}^{3} .
$$

From

$$
\Gamma_{\mu}(x)=\frac{1}{4} g_{\rho \mu}\left(\partial_{\mu} e_{\sigma}^{b} e_{a}^{\rho}-\Gamma_{\mu \sigma}^{\rho}\right) \gamma^{\delta} \gamma^{\sigma}
$$

we get

$$
\Gamma_{0}=-\frac{1}{2} \mathrm{e}^{-2 \beta} \bar{\gamma}^{0} \bar{\gamma}^{1} \gamma^{\prime}, \quad \Gamma_{1}=0, \quad \Gamma_{2}=\frac{1}{2} \mathrm{e}^{-\beta-\gamma} \bar{\gamma}^{2} \bar{\gamma}^{1} \beta^{\prime}, \quad \Gamma_{3}=\frac{1}{2} \mathrm{e}^{-\beta-\gamma} \bar{\gamma}^{3} \bar{\gamma}^{1} \beta^{\prime}
$$

The Dirac's matrices $\bar{\gamma}^{a}$ in flat space-time are chosen as in [8] [9].

Using Einstein's sommation, we find

$$
\gamma^{\mu} \Gamma_{\mu}=-\frac{1}{2} \mathrm{e}^{-\alpha} \alpha^{\prime} \bar{\gamma}^{1}
$$

Taking into account the obtained expression for $\gamma^{\mu} \Gamma_{\mu}$ (21), we can rewrite 
the Equations (9) and (10) as follows

$$
\begin{gathered}
i \mathrm{e}^{-\alpha} \bar{\gamma}^{1}\left(\partial_{x}+\frac{1}{2} \alpha^{\prime}\right) \psi-m \psi+2 i P \frac{\partial F\left(I_{p}\right)}{\partial I_{p}} \gamma^{5} \psi=0, \\
\mathrm{e}^{-\alpha}\left(\partial_{x}+\frac{1}{2} \alpha^{\prime}\right) \overline{\psi \gamma}^{1}+m \bar{\psi}-2 i P \frac{\partial F\left(I_{p}\right)}{\partial I_{p_{1}}(x)} \gamma^{5} \bar{\psi}=0 .
\end{gathered}
$$

Further setting $\psi(x)=V_{\delta}(x)$ with $\left.V_{\delta}(x)=\mid \begin{array}{l}V_{2}(x) \\ V_{3}(x)\end{array}\right]$, for the components of spinor field one deduces the following set of equations from (22)

$$
\begin{gathered}
V_{4}^{\prime}+\frac{1}{2} \alpha^{\prime} V_{4}+i m \mathrm{e}^{\alpha} V_{1}-2 P F^{\prime}\left(I_{p}\right) \mathrm{e}^{\alpha} V_{3}=0 . \\
V_{3}^{\prime}+\frac{1}{2} \alpha^{\prime} V_{3}+i m \mathrm{e}^{\alpha} V_{2}-2 P F^{\prime}\left(I_{p}\right) \mathrm{e}^{\alpha} V_{4}=0 . \\
V_{2}^{\prime}+\frac{1}{2} \alpha^{\prime} V_{2}-i m \mathrm{e}^{\alpha} V_{3}+2 P F^{\prime}\left(I_{p}\right) \mathrm{e}^{\alpha} V_{1}=0 . \\
V_{1}^{\prime}+\frac{1}{2} \alpha^{\prime} V_{1}-i m \mathrm{e}^{\alpha} V_{4}+2 P F^{\prime}\left(I_{p}\right) \mathrm{e}^{\alpha} V_{2}=0 .
\end{gathered}
$$

The functions $V_{1}, V_{2}, V_{3}$ and $V_{4}$ are connected by the relations

$$
V_{1}^{2}-V_{2}^{2}-V_{3}^{2}+V_{4}^{2}=c s t e \text { and } \bar{V}_{1} V_{4}+\bar{V}_{2} V_{3}=\bar{V}_{3} V_{2}+\bar{V}_{4} V_{1} \text {. }
$$

The set of Equations (24)-(27), leads to the system of equations for invariant functions $S=\bar{\psi} \psi, P=i \bar{\psi} \gamma^{5} \psi$ and $R=\bar{\psi}^{5} \bar{\gamma}^{1} \psi$ :

$$
\begin{gathered}
S^{\prime}+\alpha^{\prime} S+4 F^{\prime}\left(I_{p}\right) P \mathrm{e}^{\alpha} R=0 \\
R^{\prime}+\alpha^{\prime} R+2 m \mathrm{e}^{\alpha} P+4 F^{\prime}\left(I_{p}\right) P \mathrm{e}^{\alpha} S=0 \\
P^{\prime}+\alpha^{\prime} P+2 m \mathrm{e}^{\alpha} R=0
\end{gathered}
$$

Immediately, the solution of the system of equations of the invariant functions is

$$
\begin{gathered}
P^{2}-R^{2}+S^{2}=C \mathrm{e}^{-2 \alpha(x)}, \\
C=\text { const } .
\end{gathered}
$$

Using the spinor field equation in the form (9) and the conjugate one in the form (10), we obtain the following expression for $T_{1}^{1}$ from (15):

$$
T_{1}^{1}=m S-F\left(I_{p}\right)
$$

Let us now solve the Einstein equation. In view of $T_{0}^{0}=T_{2}^{2}$, the difference $\left(\begin{array}{l}0 \\ 0\end{array}\right)-\left(\begin{array}{l}2 \\ 2\end{array}\right)$ of the Einstein equations leads to

$$
\beta^{\prime \prime}-\gamma^{\prime \prime}=0
$$

By integrating, the Equation (34) has the solution

$$
\beta(x)=\gamma(x)+A x
$$

where $A$ is the integration constant; another integration constant has been chosen equal to zero since the functions $\beta$ and $\gamma$ depend only of the spatial 
variable $x$.

From (3), we have

$$
\alpha^{\prime \prime}=2 \beta^{\prime \prime}+\gamma^{\prime \prime}
$$

Taking into account (34), we obtain from (36) the following equalities

$$
\beta^{\prime \prime}(x)=\frac{1}{3} \alpha^{\prime \prime}(x), \quad \gamma^{\prime \prime}(x)=\frac{1}{3} \alpha^{\prime \prime}(x) .
$$

The solutions to the Equation (37) using (3) and (35) lead to the following expressions for $\beta(x)$ and $\gamma(x)$ :

$$
\beta(x)=\frac{1}{3}(\alpha(x)+B x), \quad \gamma(x)=\frac{1}{3}(\alpha(x)-2 B x)
$$

where $B$ is the integration constant. Taking into account the expressions (35) and (38), we deduce easily $A=B$.

The Equation (6) being the first integral of (5) and (7), is a first order differential equation. Inserting (38) into (5) and substituting the result into (33), we get the Eintein equation $\left(\begin{array}{l}1 \\ 1\end{array}\right)$ under the form

$$
\left(\alpha^{\prime}\right)^{2}-A^{2}=-3 \chi \mathrm{e}^{2 \alpha}\left[m S-F\left(I_{p}\right)\right] .
$$

In order to solve the Equation (39), it is necessary to choose massless spinor field, i.e., $m=0$ according to unified nonlinear spinor theory of Heisenberg. The mass term should be obtained after quantization. For details, refer to [10] [11] and references therein.

From (31), for $m=0, \alpha^{\prime}=-\frac{1}{P} \frac{\mathrm{d} P}{\mathrm{~d} x}$. Therefore (39) becomes

$$
\frac{\mathrm{d} P}{\sqrt{A^{2} P^{2}+3 \chi C_{0}^{2} F\left(I_{p}\right)}}= \pm \mathrm{d} x .
$$

Thus, the general exact solutions of Einstein equations and nonlinear spinor field equations are:

$$
\int \frac{\mathrm{d} P}{\sqrt{A^{2} P^{2}+3 \chi C_{0}^{2} F\left(I_{p}\right)}}= \pm\left(x+x_{0}\right), \quad x_{0}=\text { cste. }
$$

Let us remark that the general solutions depend on the analytics explicits forms of $F\left(I_{p}\right)$. Indeed knowing $F\left(I_{p}\right)$, we can find $P(x)$ from (41). If $P(x)$ is known, we can determine $\alpha(x)$ from (31) and if $\alpha(x)$ is known, we can determine easily $\beta(x)$ and $\gamma(x)$ from (38).

Taking into account $P(x)=C_{0} \mathrm{e}^{-\alpha(x)}$, we can establish the regular properties of the obtained solutions and on the other hand to establish the regularity of the metric functions and the matter fields in the whole space-time. Studying the energy density $T_{0}^{0}$, we can establish the localization properties of the solutions and the finiteness of The total energy [2] [8].

Let us resolve the spinor field equations in the following paragraph. We can get a concrete form of the functions $V_{\delta}$ by solving Equations (24)-(27) in a 
more compacte form if we pass to the functions $V_{\delta}=\mathrm{e}^{-\frac{\alpha}{2}} W_{\delta}, \delta=1,2,3,4$ :

$$
\begin{aligned}
& W_{4}^{\prime}(x)-\phi(x) W_{3}(x)=0 \\
& W_{3}^{\prime}(x)-\phi(x) W_{4}(x)=0 \\
& W_{2}^{\prime}(x)+\phi(x) W_{1}(x)=0 \\
& W_{1}^{\prime}(x)+\phi(x) W_{2}(x)=0
\end{aligned}
$$

With $\phi(x)=2 F^{\prime}\left(I_{p}\right) P \mathrm{e}^{\alpha}$. Let us find from the set of first-order Equations (42)-(45) to a set second-order equations for the functions $W_{1}, W_{2}, W_{3}, W_{4}$.

We have:

$$
\begin{aligned}
& W_{1}^{\prime \prime}-\frac{\phi^{\prime}}{\phi} W_{1}^{\prime}-\phi^{2} W_{1}=0 \\
& W_{2}^{\prime \prime}-\frac{\phi^{\prime}}{\phi} W_{2}^{\prime}-\phi^{2} W_{2}=0 \\
& W_{3}^{\prime \prime}-\frac{\phi^{\prime}}{\phi} W_{3}^{\prime}-\phi^{2} W_{3}=0 \\
& W_{4}^{\prime \prime}-\frac{\phi^{\prime}}{\phi} W_{4}^{\prime}-\phi^{2} W_{4}=0
\end{aligned}
$$

The solution of the Equation (46) is

$$
W_{1}(x)=C_{1} \exp \left(\int \phi(x) \mathrm{d} x\right)+i C_{2} \exp \left(-\int \phi(x) \mathrm{d} x\right)
$$

where $C_{1}$ and $C_{2}$ are integration constants.

From (45), the solution of the Equation (47) is

$$
W_{2}(x)=-C_{1} \exp \left(\int \phi(x) \mathrm{d} x\right)+i C_{2} \exp \left(-\int \phi(x) \mathrm{d} x\right) .
$$

Solving analogously Equations (48) and (49), we obtain the following expressions for $W_{3}$ and $W_{4}$ :

$$
\begin{aligned}
& W_{3}(x)=C_{3} \exp \left(\int \phi(x) \mathrm{d} x\right)+i C_{4} \exp \left(-\int \phi(x) \mathrm{d} x\right), \\
& W_{4}(x)=C_{3} \exp \left(\int \phi(x) \mathrm{d} x\right)-i C_{4} \exp \left(-\int \phi(x) \mathrm{d} x\right)
\end{aligned}
$$

with $C_{3}$ and $C_{4}$ are integration constants.

Let us determine the link between the integration constants $C, C_{0}, C_{1}, C_{2}$, $C_{3}$ and $C_{4}$ which are in the solutions of Einstein's and spinor fields equations.

From $V_{\delta}=\mathrm{e}^{-\frac{\alpha}{2}} W_{\delta}$, we get

$$
\begin{gathered}
P=4\left(C_{1} C_{4}-C_{2} C_{3}\right) \exp (-\alpha) \\
R(x)=2\left[\left(C_{2}^{2}-C_{4}^{2}\right) \exp \left(-2 \int \phi(x) \mathrm{d} x\right)+\left(C_{3}^{2}-C_{1}^{2}\right) \exp \left(2 \int \phi(x) \mathrm{d} x\right)\right] \exp (-\alpha), \\
S(x)=2\left[\left(C_{1}^{2}-C_{3}^{2}\right) \exp \left(2 \int \phi(x) \mathrm{d} x\right)+\left(C_{2}^{2}-C_{4}^{2}\right) \exp \left(2 \int \phi(x) \mathrm{d} x\right)\right] \exp (-\alpha) .
\end{gathered}
$$

Substituting the expressions (50), (51), (52) and (53) obtained previously in (32), we have 


$$
C=16\left(C_{1} C_{2}-C_{3} C_{4}\right)^{2} .
$$

Later, comparing the expression of $P(x)$ in (54) and $P(x)=C_{0} \mathrm{e}^{-\alpha}$, we have

$$
C_{0}=4\left(C_{1} C_{4}-C_{2} C_{3}\right) \text {. }
$$

Thus, we have obtained the general solutions of the Equations (46)-(49) which contain four arbitrary constants $C_{1}, C_{2}, C_{3}$ and $C_{4}$.

In the following section, we shall analyze the general solutions obtained in the previous section choosing the nonlinear term in the concrete form.

\section{Analysis of the Results: Concrete Form of Nonlinear Term in Spinor Lagrangian}

Let us study the solution to linear Equation (Dirac's equation). Note that this solution is necessary for comparing with solutions to nonlinear spinor equations in order to clarify the influence of nonlinear terms in the nonlinear field equations in the formation of configuation of elementary particles.

In the linear case $L_{N}=0$ for $m=0$ we have from (22)

$$
i \mathrm{e}^{-\alpha} \bar{\gamma}^{1}\left(\partial_{x}+\frac{1}{2} \alpha^{\prime}\right) \psi=0
$$

Taking into account (41), we get

$$
P(x)=C_{0} \mathrm{e}^{-A\left(x+x_{0}\right)}
$$

As $P(x)=C_{0} \mathrm{e}^{-\alpha}$, from (60), we obtain the expression of the metric function:

$$
\alpha(x)=A\left(x+x_{0}\right)
$$

From (38), we find the following expressions of $\beta$ and $\gamma$ :

$$
\begin{aligned}
& \beta(x)=\frac{2}{3} A\left(x+x_{0}\right) \\
& \gamma(x)=-\frac{1}{3} A\left(x+x_{0}\right)
\end{aligned}
$$

With $L_{N}=0$, the energy density is

$$
T_{0}^{0}=0
$$

From (64) it follows that, the energy density of the system is not localized. In sum, the soliton-like solutions are absent in the linear case.

Let us now consider a concrete type of nonlinear spinor field equation in the form $L_{N}=F\left(I_{p}\right)=\lambda P^{2 n}$ where $\lambda$ is a nonlinearity parameter and $n$ is a power of nonlinearity. In the first case, For $n=1$, we find Heisenberg-Ivanenko type nonlinear spinor field equation [10]

$$
i e^{-\alpha} \bar{\gamma}^{1}\left(\partial_{x}+\frac{1}{2} \alpha^{\prime}\right) \psi-m \psi+2 i P \lambda \gamma^{5} \psi=0,
$$

Substituting $F\left(I_{P}\right)=\lambda I_{P}=\lambda P^{2}$ into (41), we obtain the similar expression as for a linear spinor field, only now the constant $A^{2}$ is replaced by $A^{2}+3 \chi \lambda C_{0}^{2}$. 
We have

$$
P(x)=C_{0} \mathrm{e}^{\left(x+x_{0}\right) \sqrt{A^{2}+3 \chi \lambda C_{0}^{2}}}
$$

From the relation $P=C_{0} \mathrm{e}^{-\alpha}$, we get

$$
\alpha(x)=-\left(x+x_{0}\right) \sqrt{A^{2}+3 \chi \lambda C_{0}^{2}} .
$$

According to the equality (38), we define the functions $\beta(x)$ and $\gamma(x)$ as follows

$$
\begin{aligned}
& \beta(x)=\frac{1}{3}\left[-\left(x+x_{0}\right) \sqrt{A^{2}+3 \chi \lambda C_{0}^{2}}+A x\right] \\
& \gamma(x)=-\frac{1}{3}\left[\left(x+x_{0}\right) \sqrt{A^{2}+3 \chi \lambda C_{0}^{2}}+2 A x\right]
\end{aligned}
$$

The invariant $I_{P}=P^{2}$ and the functions $g_{00}=\mathrm{e}^{2 \gamma}, g_{11}=-\mathrm{e}^{2 \alpha}$, $g_{22}=g_{33}=-\mathrm{e}^{2 \beta}$ are regular in all space.

The energy density $T_{0}^{0}$ has the following expression:

$$
T_{0}^{0}=C_{0}^{2} \mathrm{e}^{2\left(x+x_{0}\right) \sqrt{A^{2}+3 \chi \lambda C_{0}^{2}}}
$$

hence we obtain for $\varepsilon(x)$ the energy density per unit invariant volume

$$
\varepsilon(x)=T_{0}^{0} \sqrt{-3_{g}}=C_{0}^{2} \mathrm{e}^{2\left(x+x_{0}\right) \sqrt{A^{2}+3 x \lambda C_{0}^{2}}} \mathrm{e}^{\frac{2}{3} A x}
$$

We conclude from (70) and (71) that a localization of the energy density and the energy density per unit invariant volume are absent. The total energy diverges. The Equation (65) has no soliton-like solution.

In the second case when $F\left(I_{P}\right)=\lambda P^{2 n}$ and $n>1$, From (41), we have

$$
P(x)=\left\{\frac{A}{\sqrt{3 \chi \lambda C_{0}^{2}} \sinh [A(n-1) x]}\right\}^{\frac{1}{n-1}} ; n>1 .
$$

From (14), the energy density is

$$
T_{0}^{0}=\lambda(2 n-1)\left\{\frac{A}{\sqrt{3 \chi \lambda C_{0}^{2}} \sinh [A(n-1) x]}\right\}^{\frac{2 n}{n-1}} ; n>1 .
$$

We remark that, from $L_{N}=\lambda P^{2 n} ;(72)$ and (73), when $x \rightarrow 0, P(x) \rightarrow \infty$ and $T_{0}^{0} \rightarrow \infty$.

It follows that the energy density is not localized and the total energy does not finite value.

When $\lambda=-\Lambda^{2}$, from the relation (41), we deduce that:

$$
P(x)=\left\{\frac{A}{\sqrt{3 \chi \Lambda^{2} C_{0}^{2}} \cosh [A(n-1) x]}\right\}^{\frac{1}{n-1}}
$$

From (74), it follows that $P(x)$ has its maximum value $\left\{\frac{A}{\sqrt{3 \chi \Lambda^{2} C_{0}^{2}}}\right\}^{\frac{1}{n-1}}$; 
$n>1$ when $x=0$ and $P(x) \rightarrow 0$ when $x \rightarrow \pm \infty$. In this case $T_{0}^{0}$ is

$$
T_{0}^{0}=-\Lambda^{2}(2 n-1)\left\{\frac{A}{\sqrt{3 \chi \Lambda^{2} C_{0}^{2}} \cosh [A(n-1) x]}\right\}^{\frac{2 n}{n-1}} ; n>1
$$

From the expression (75), it follows that the energy density $T_{0}^{0}$ of a nonlinear spinor field is negative and localized in space when the nonlinear term of the equation is chosen under the form $L_{N}=F\left(I_{p}\right)=-\Lambda^{2} P^{2 n}$ with $-\Lambda^{2}<0$ [2] [12] [13].

The energy density per unit invariant volume is:

$$
\varepsilon(x)=T_{0}^{0} \sqrt{-3_{g}}=-\Lambda^{2}(2 n-1) C_{0}^{\frac{5}{3}}\left\{\frac{A}{\sqrt{3 \chi C_{0}^{2} \Lambda^{2}} \cosh [A(n-1) x]}\right\}^{\frac{-4 n}{3(n-1)}} \mathrm{e}^{\frac{2}{3} A x}
$$

From (76), it follows that the energy density is negative and localized. Indeed, when $A>0 ; n>1$ and for $x \rightarrow \pm \infty ; \varepsilon \rightarrow 0$. Furthermore, $\varepsilon(x)$ has its minimal value $-\Lambda^{2}(2 n-1) C_{0}^{\frac{5}{3}}\left\{\frac{A}{\sqrt{3 \chi C_{0}^{2} \Lambda^{2}}}\right\}^{\frac{-4 n}{3(n-1)}}$ when $x=0$.

The total energy of spinor field is defined by

$$
E_{f}=\int_{-\infty}^{+\infty} \varepsilon(x) \mathrm{d} x=\int_{-\infty}^{+\infty} T_{0}^{0} \sqrt{-3_{g}} \mathrm{~d} x .
$$

Thus

$$
E_{f}=\int_{-\infty}^{+\infty}-\Lambda^{2}(2 n-1) C_{0}^{\frac{5}{3}}\left\{\frac{A}{\sqrt{3 \chi C_{0}^{2} \Lambda^{2}} \cosh [A(n-1) x]}\right\}^{\frac{-4 n}{3(n-1)}} \mathrm{e}^{\frac{2}{3} A x} \mathrm{~d} x<\infty .
$$

$\varepsilon(x)$ is a regular, negative and localized function therefore the total energy $E_{f}$ is limited and negative.

Since the obtained solution is regular and has a localized energy density $T_{0}^{0}$, the total energy $E_{f}$ negative, limited and the metric functions regular and limited, it is a soliton-like solution. Moreover, this solution can be used to describe the configuration of the elementary particles.

From (50)-(53), we can obtain the concrete form of the functions $V_{\delta}(x)$ in their more compact form.

$$
\begin{aligned}
& V_{1}(x)=\left(C_{1} \mathrm{e}^{\sigma(x)}+i C_{2} \mathrm{e}^{-\sigma(x)}\right) \cdot \mathrm{e}^{-\frac{\alpha}{2}} \cdot \\
& V_{2}(x)=\left(-C_{1} \mathrm{e}^{\sigma(x)}+i C_{2} \mathrm{e}^{-\sigma(x)}\right) \cdot \mathrm{e}^{-\frac{\alpha}{2}} . \\
& V_{3}(x)=\left(C_{3} \mathrm{e}^{\sigma(x)}+i C_{4} \mathrm{e}^{-\sigma(x)}\right) \cdot \mathrm{e}^{-\frac{\alpha}{2}} . \\
& V_{4}(x)=\left(C_{3} \mathrm{e}^{\sigma(x)}-i C_{4} \mathrm{e}^{-\sigma(x)}\right) \cdot \mathrm{e}^{-\frac{\alpha}{2}} .
\end{aligned}
$$

where $C_{1} ; C_{2} ; C_{3}$ and $C_{4}$ are integration constants. Furtermore, 
$\mathrm{e}^{-\frac{\alpha}{2}}=\left[P(x) / C_{0}\right]^{\frac{1}{2}}$ with $P(x)$ defined by the expression (74).

$$
\sigma(x)=\int \Phi(x) \mathrm{d} x=2 \int F^{\prime}\left(I_{P}\right) C_{0} \mathrm{~d} x=-\frac{2 n A}{3 \chi C_{0}(n-1)} \tanh [A(n-1) x]
$$

$P(x)$ is a regular function, then $\sigma(x)$ and $V_{\delta}(x)$ are also the regular functions.

In sum, for a massless field (i.e. $m=0$ ) and $F\left(I_{p}\right)=-\Lambda^{2} I_{p}^{n} ; n>1$; the solutions of the equations of the spinor and gravitational fields have an energy density $\varepsilon(x)$ and total energy $E_{f}$ negative, regular and localized.

Let us now study the influence of the proper gravitational field in the formation of the configuration of elementary particles.

In order to determine the role of the own gravitational field in the formation of regular localized solutions of soliton-like type to nonlinear spinor field equations, it is necessary to consider solutions to Equation (9) in flat space-time when $\beta=\alpha=\gamma=0$ in (2). In this case, from (41) we get

$$
P=C_{0}=\text { cste. }
$$

The explicit form of $V_{\delta}(x)$ is:

$$
\begin{aligned}
& V_{1}(x)=\left(C_{1} \mathrm{e}^{M x}+i C_{2} \mathrm{e}^{-M x}\right) . \\
& V_{2}(x)=\left(-C_{1} \mathrm{e}^{M x}+i C_{2} \mathrm{e}^{-M x}\right) . \\
& V_{3}(x)=\left(C_{3} \mathrm{e}^{M x}+i C_{4} \mathrm{e}^{-M x}\right) . \\
& V_{4}(x)=\left(C_{3} \mathrm{e}^{M x}-i C_{4} \mathrm{e}^{-M x}\right) .
\end{aligned}
$$

where $\sigma(x)=M x ; M=$ cste and $T_{0}^{0}=$ cste.

We deduce that, the proper gravitational field of elementary particles plays an important role in the aim to obtain the regular solutions which possess a localized energy density and limited total energy of the nonlinear equations with the nonlinear term depending on $P^{2}$.

The last point is addressed to the spinor current vector and the total charge. Using the general solutions of the Equations (24)-(27), we can write the components of the spinor current vector $j^{\mu}=\bar{\psi} \gamma^{\mu} \psi$ :

$$
\begin{gathered}
j^{0}=\psi^{+} \bar{\gamma}^{0} \gamma^{0} \psi \\
j^{0}=\left(V_{1}^{*} V_{1}+V_{2}^{*} V_{2}+V_{3}^{*} V_{3}+V_{4}^{*} V_{4}\right) \mathrm{e}^{-\alpha-\gamma} . \\
j^{0}=\left[C_{1}^{2} \cosh ^{2} \sigma(x)+C_{2}^{2} \cosh ^{2} \sigma(x)+C_{1}^{2} \sinh ^{2} \sigma(x)+C_{2}^{2} \sinh ^{2} \sigma(x)\right] \mathrm{e}^{-\alpha-\gamma} . \\
j^{1}=\psi^{+} \bar{\gamma}^{0} \gamma^{1} \psi \\
j^{1}=\left[V_{1}^{*} V_{4}+V_{2}^{*} V_{3}+V_{3}^{*} V_{2}+V_{4}^{*} V_{1}\right] \mathrm{e}^{-2 \alpha} . \\
j^{1}=0 . \\
j^{2}=\psi^{+} \bar{\gamma}^{0} \gamma^{2} \psi \\
j^{2}=i\left[V_{1}^{*} V_{4}+V_{2}^{*} V_{3}+V_{3}^{*} V_{2}+V_{4}^{*} V_{1}\right] \mathrm{e}^{-2 \alpha} .
\end{gathered}
$$




$$
\begin{gathered}
j^{2}=-2\left[C_{1}^{2} \cosh \sigma(x) \sinh (x)-C_{2}^{2} \cosh \sigma(x) \sinh \sigma(x)\right] \mathrm{e}^{-\alpha-\beta} . \\
j^{3}=\psi^{+} \bar{\gamma}^{0} \gamma^{3} \psi \\
j^{3}=\left[V_{1}^{*} V_{3}-V_{2}^{*} V_{4}+V_{3}^{*} V_{1}-V_{4}^{*} V_{2}\right] \mathrm{e}^{-\alpha-\gamma} . \\
j^{3}=0 .
\end{gathered}
$$

Since the field configuration is chosen to be static plane symmetric, the spatial components of the spinor current vanishes. Alone the component $j^{0}$ is nonzero. This assumption leads to the following relations between the constants:

$$
j^{1}=j^{2}=j^{3}=0 \text { and } j^{2}=0 \Rightarrow C_{1}=C_{2}=a .
$$

The component $j^{0}$ defines the charge density of spinor field that has the following chronometric-invariant form given by the expression

$$
\rho(x)=\left(j_{0} j^{0}\right)^{\frac{1}{2}}=2 a^{2} \cosh [2 \sigma(x)] \mathrm{e}^{-\alpha} .
$$

The total charge of the spin is defined by

$$
Q=\int_{-\infty}^{+\infty} \rho(x) \sqrt{3_{-g}} \mathrm{~d} x
$$

The spin tensor is given by the expression as in [9] [11]

$$
S^{\mu v, \lambda}=\frac{1}{4} \bar{\psi}\left\{\gamma^{\lambda} \sigma^{\mu \nu}+\sigma^{\mu v} \gamma^{\lambda}\right\} \psi
$$

The spatial density of the spin tensor $S^{i k, 0}, i, k=1 ; 2 ; 3$ from (95) is:

$$
S^{i k, 0}=\frac{1}{4} \bar{\psi}\left\{\gamma^{0} \sigma^{i k}+\sigma^{i k} \gamma^{0}\right\} \psi=\frac{1}{2} \bar{\psi} \gamma^{0} \sigma^{i k} \psi
$$

Here $\mathrm{i}, \mathrm{j}, \mathrm{k}$ take the value $1,2,3$ and $i \neq j \neq k$. Thus, for the projection of spin vector on the $\mathrm{x}, \mathrm{y}$ and $\mathrm{z}$ axis, we get:

$$
\begin{aligned}
S^{23,0} & =\left[V_{1}^{*} V_{2}+V_{2}^{*} V_{1}+V_{3}^{*} V_{4}+V_{4}^{*} V_{3}\right] \mathrm{e}^{-\alpha-2 \beta-\gamma} \\
S^{31,0} & =\left[V_{1}^{*} V_{2}-V_{2}^{*} V_{1}+V_{3}^{*} V_{4}-V_{4}^{*} V_{3}\right] \mathrm{e}^{-2 \alpha-\beta-\gamma} \\
S^{12,0} & =\left[V_{1}^{*} V_{1}-V_{2}^{*} V_{2}+V_{3}^{*} V_{3}+V_{4}^{*} V_{4}\right] \mathrm{e}^{-2 \alpha-\beta-\gamma}
\end{aligned}
$$

Thus, we have

$$
\begin{gathered}
S^{12,0}=S^{13,0}=0 . \\
S^{23,0}=a^{2} \cosh [2 \sigma(x)] \mathrm{e}^{-\alpha} .
\end{gathered}
$$

From (101) the chronometric invariant spin tensor is defined by:

$$
S_{c h l}^{23,0}=\left(S_{23,0} S^{23,0}\right)^{\frac{1}{2}}=a^{2} \cosh [2 \sigma(x)] \mathrm{e}^{-\alpha} .
$$

We remark that the total charge and total spin are not limited because from (93), $\rho(x)$ is not a localized function since when $x \rightarrow \pm \infty$, we have $\rho(x) \rightarrow \pm \infty$. Thus, we can note that the nonlinearity of the spinor field equation and the consideration of the proper gravitational field of the elementary particles are the necessary conditions but not sufficient in order to obtain the limited total charge and total spin to confirm what are got experimentaly. 


\section{Concluding Remarks}

In this research work, we have obtained the analytic general solutions of nonlinear spinor and gravitational fields equations which are regular, localized energy density and finite total energy. These solutions are soliton like solutions. They can be used in order to describe the configuration of elementary particles. In flat space-time and linear cases, the soliton like solutions are absent. The role of the own gravitational field is crucial for the formation of soliton-like configuration of nonlinear spinor fields. In the case of the study of polynomial nonlinearities $L_{N}=\lambda P^{2 n}, n>1$, we remark that the obtained solutions are regular, localized energy density and finite quantity of total energy in the exclusively case where $\lambda=-\Lambda^{2}$. Emphasize that the total charge and total spin are not limited in the all metric case. Our perspective, in the forthcomming research work, is to examine the spherical symmetric soliton-like solutions to the spinor field equations with nonlinear terms $L_{N}=F\left(I_{P}\right)$ which are arbitrary functions of $I_{P}=\left(i \bar{\psi} \gamma^{5} \psi\right)^{2}$.

\section{Conflicts of Interest}

The authors declare no conflicts of interest regarding the publication of this paper.

\section{References}

[1] Shikin, G.N. (1995) Theory of Solitons in General Relativity. URSS, Moscow.

[2] Adomou, A. and Shikin, G.N. (1998) Izvestia VUZov, Fizika, 41, 69.

[3] Saha, B. and Shikin, G.N. (2003) Czechoslovak Journal of Physics, 54, 597-620. https://doi.org/10.1023/B:CJOP.0000029690.61308.a5

[4] Adanhoum, A., Adomou, A., Codo, F.P. and Hounkonnou, M.N. (2012) Journal of Modern Physics, 3, 935. https://doi.org/10.4236/jmp.2012.39122

[5] Brill, D. and Wheeler, J. (1957) Reviews of Modern Physics, 29, 465. https://doi.org/10.1103/RevModPhys.29.465

[6] Zhelnorovich, V.A. (1982) Spinor Theory and Its Applications in Physics and Mechanics. Nauka, Moscow.

[7] Saha, B. and Shikin, G.N. (1996) International Journal of Modern Physics A, 15, 1481. https://doi.org/10.1016/S0217-751X(00)00066-5

[8] Adomou, A., Alvarado, R. and Shikin, G.N. (1995) Izvestiya Vuzov. Fizika, 863-868.

[9] Bogoliubov, N.N. and Shirkov, D.V. (1976) Introduction to the Theory of Quantized Fields. Nauka, Moscow.

[10] Heisenberg, W. (1966) Introduction to Unified Field Theory of Elementary Particles. Interscience Publishers, London.

[11] Schweber, S. (1961) Introduction to Relativistic Quantum Field Theory. Harper and Row, New York.

[12] Weyl, H. (1950) Physical Review, 77, 699-701.

[13] Rybakov, Yu.P., Shikin, G.N. and Saha, B. (1997) International Journal of Theoretical Physics, 36, 1475-1494. https://doi.org/10.1007/BF02435941 